\title{
Analysis of Ultrasonic Pancreatic Histograms in Diabetes Mellitus
}

\author{
Shigeki Enomoto \\ The Third Department of Internal Medicine, Kansai Medical University, \\ Moriguchi, Osaka 570, Japan \\ (Supervised by Prof. Y. Sameshima) \\ (Received for publication November 30, 1989)
}

Key words : Ultrasound Histogram, Diabetes Mellitus, Pancreas

\begin{abstract}
Problems have been involved in the sonographical diagnosis of diffuse pancreatic and hepatic diseases in terms of its quantitativity and objectivity, because the diagnosis relies highly on the examiner's subjective judgement.

This study was attempted for a easier and more objective assessment on the sonographical changes in the pancreas of the diabetics by analysing the histograms of echoic levels and pixel distribution.

Fifty six patients with their age nearly equally distributed from 10 through 89 years with impaired glucose tolerance were subjected to the clinical study, along with 112 healthy controls of the similar age distribution. Sixteen other cases diagnosed as having diabetes mellitus both clinically and histologically and 13 controls having healthy pancreas and liver were chosen for the postmortem study. A $3.5 \mathrm{MHz}$ transducer was used for both studies.

A region of interest (ROI) of a square centimeter was designated on sagittal scanning in the pancreas body and in the liver at the same depth from the body surface in the clinical study. Water immersion technique was employed for the postmortem study. To avoid multiple reflection, the ROI was placed inside the pancreas and the liver within the depth of $3 \mathrm{~cm}$ below the water surface.

A ROI consists of 3600 pixels and the luminance of pixels can be devided into 64 levels. The level of luminance having [the maximum number of pixels is given by " $L$ " and the number of pixels is shown by " $\mathrm{M}$ " or the histogram at the $\mathrm{L}$ on the visual display. $\mathrm{L}$ : pancreas/liver (1), L : pancreas-liver (2), M : pancreas/liver (3) and M : pancreas-liver (4) were then calculated from the data displayed.
\end{abstract}

Among the healthy controls, both (1) and (2) were significantly bigger in the group over 70 years of age than in those of the remaining age categories. The postmortem histology 
showed that the age-related change was due to degeneration and irregularity of acinar structure accompanied by fibrosis.

Among the diabetics, (3) and (4) were significantly lower, compared with those of the healthy controls. Histologically, the decrease was associated with degeneration (hyalinosis) of the islets.

\section{Purpose}

It has been reported that ultrasonic diagnosis of diffuse diseases in abdominal organs such as the liver and pancreas involves problems as to quantitativity and objectivity because it relies highly on the subjective judgment of the examiner.

Quantitative determination of acoustic characteristics of tissue have been performed by : (1) determination of frequency-dependent attenuation ${ }^{1-5)}$; (2) histogram analysis using B-mode ultrasound images ${ }^{6-14)}$; and (3) determination of acoustic velocity ${ }^{15,16)}$.

Although the clinical use of these three methods employing removed tissue specimens is gradually increasing, they require complicated instruments and hence have not become widespread. In acoustic tissue characterization via the body surface, the intervention of subcutaneous fat and muscles affects the attenuation and scattering of sound waves, making accurate quantitation difficult ${ }^{17-19)}$.

Bearing this in mind, I recently attempted simple and objective assessment of pancreatic acoustic changes in diabetics by analyzing histograms of echo level and pixel distribution.

\section{Subjects}

1) Clinical cases

Fifty six patients (with ages homogenously distributed between 10 to 89 ) in whom a $75 \mathrm{~g}$ oral glucose tolerance test revealed abnormal findings were subjected to the study. In addition, 112 patients having healthy pancreas and liver, with a similar age distribution were selected as controls. Subjects were divided into four groups according to their age: Groups I (10-29 years), II (30-49 years), III (50-69 years) and IV (70-89 years).

2) Autopsied cases

Of the approximate 1000 cases autopsied over the past 5 years, the following 29 cases were selected: 16 cases which were clinically diagnosed as having diabetes and histologically rated as diabetes mellitus, and 13 cases with normal pancreas (controls). The pancreas removed from each of the 29 subjects was examined. All subjects had neither clinical abnormalities of hepatic function nor histological abnormalities of the liver. 


\section{Instruments}

For this study, an Aloka SSD-650 (with a histogram displaying function) and a $3.5 \mathrm{MHz}$ linear transducer were used.

\section{Methods}

\section{1) Clinlcal cases}

On the sagittal abdominal scaning a region of interest (ROI) with dimensions $1.0 \times 1.0 \mathrm{~cm}$ was designated. A similar ROI was also designated in the liver at the same depth as above. In this way, the internal echo histogram of the pancreas relative to that of the liver at the same depth image was analyzed, taking into consideration ultrasonic attenuation and scattering which can occur in ultrasound examination via body surface (Fig. 1). Cases in which the digestive tract was interposed between the pancreas and liver were excluded from this examination.

\section{2) Autopsied cases}

Water immersion echo technique was employed for the autopsied cases. To avoid multiple relection of the removed pancreas in water, the ROI was placed inside the pancreas within the depth of $3 \mathrm{~cm}$ below the water surface, and histological changes were examined in addition to analysis of histograms.

In histological examination, particular attention was paid to fibrosis, fatty infiltration,

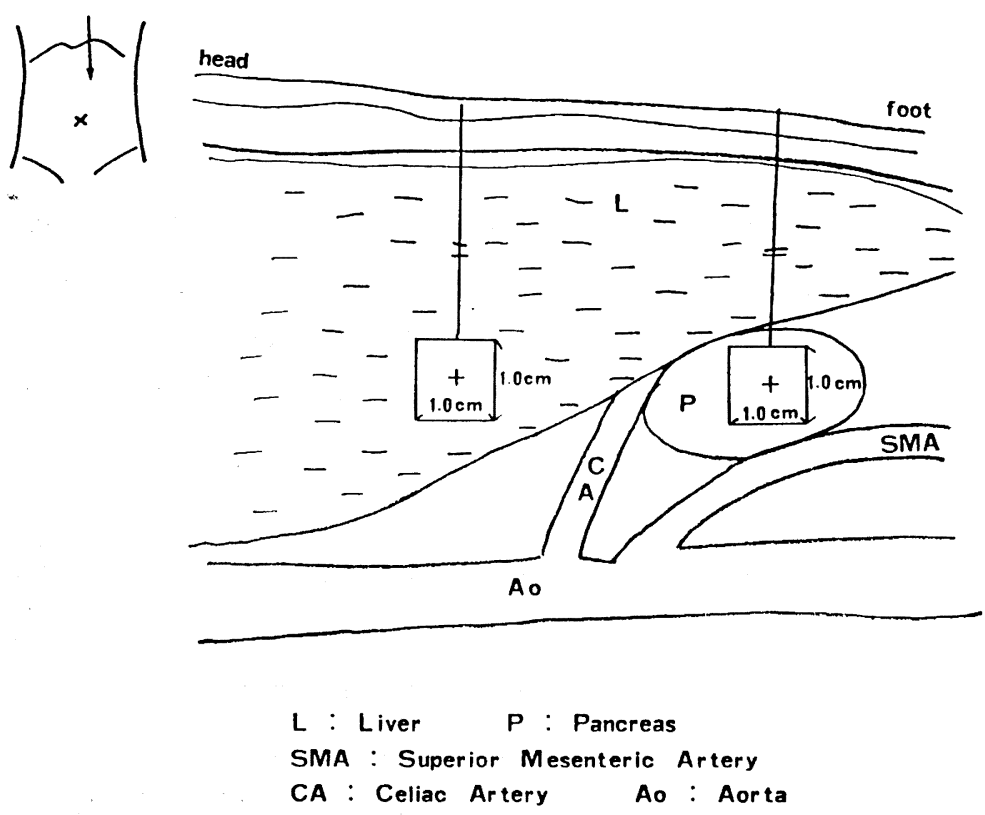

Fig. 1 Ilustration of the measurement 
disturbed acinar structure due to decrease of cellular components, and degeneration of the islet. A histogram was also obtained for the removed liver (control) in a similar manner.

\section{Determination}

One ROI on the display contains 3600 pixels. The luminace of pixels can be devided into 64 levels ( $\mathrm{O}$ to 63). The most frequent level of luminance is displayed as $\mathrm{L}$ (Level) on the monitor screen together with a histogram.

The number of pixels for $\mathrm{L}$ is simultaneously displayed as $\mathrm{M}$ (Maximum), as shown in

Fig. 2. In this way, the most frequent level of luminance in an ROI can be analyzed in relation to the number of pixels. No sensitivity time control (STC) was used, and this STC was regarded as certain in this study.

However, total gain was changed to allow the possibility of no luminance insufficiency with the influence of intervening tissue (such as subcutaneous fat) and so that luminance range could be utilized for histograms with a good balance. In analysis of reproducibility in the same cases, high reproducibility of the luminance level and pixel number was disclosed when mean values for several portions were compared. In autopsied cases, total gain was kept hardly changed because attenuation did not need to be considered in the water immersion method.

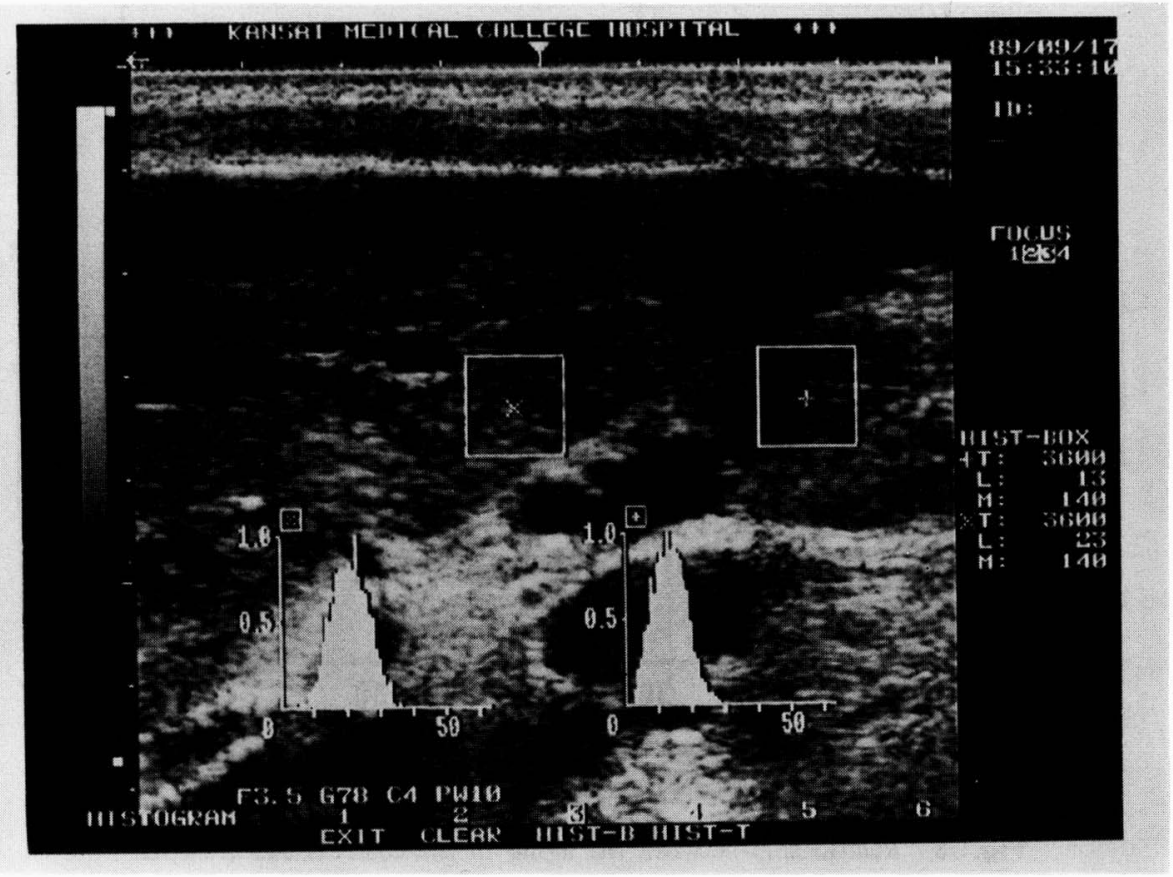

Fig. 2 An example of the histogram 
This study was calculated the retio of pancreatic $\mathrm{L}$ to hepatic $\mathrm{L}(\mathrm{L}: \mathrm{P} / \mathrm{L})$, the difference between pancreatic $\mathrm{L}$ and hepatic $\mathrm{L}(\mathrm{L}: \mathrm{P}-\mathrm{L})$, the ratio of pancreatic $\mathrm{M}$ to hepatic $\mathrm{M}(\mathrm{M}: \mathrm{P} / \mathrm{L})$ and the difference between pancreatic $M$ and hepatic $M(M: P-L)$. In clinical cases, the statistical significance of differences between diabetes qroup and controls was tested, taking into consideration aging-induced changes.

\section{Results}

1) Clinical cases

i) Influence of aging on normal pancreas

In normal pancreas, $\mathrm{L}: \mathrm{P} / \mathrm{L}$ and $\mathrm{L}: \mathrm{P}-\mathrm{L}$ increased with age. There was a positive relationship between $\mathrm{L}: \mathrm{P} / \mathrm{L}$ and age $(\mathrm{r}=0.302, \mathrm{P}<0.01)$ and between $\mathrm{L}: \mathrm{P}-\mathrm{L}$ and age $(\mathrm{r}=0.371$, $\mathrm{P}<0.01$ ). Thus, the most frequent level of luminance, indicative of the pancreatic echo level, was elevated with age (Fig. 3, 4).

In comparison between different age groups (divided at intervals of 20 years), the most frequent level was significantly higher in Group IV (L:P/L=1.79 $\pm 0.12 ; \mathrm{L}: \mathrm{P}-\mathrm{L}=10.86$ $\pm 1.06)$ than in the other age groups (Fig. 5).

In terms of pixel counts $(\mathrm{M}: \mathrm{P} / \mathrm{L}$ and $\mathrm{M}: \mathrm{P}-\mathrm{L})$, no significant inter-group difference was noted.

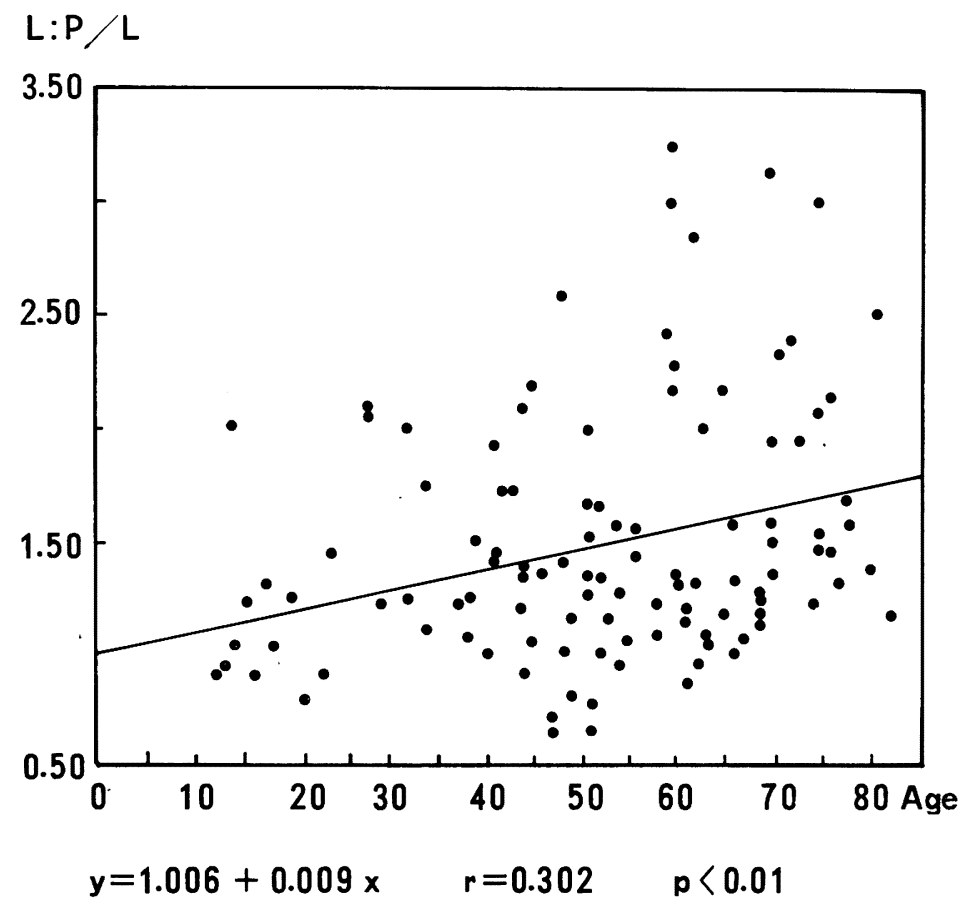

Fig. 3 Relationship between the aging in the controls and $\mathrm{L}: \mathrm{P} / \mathrm{L}$ value of the echogenicity index 
$L: P-L$

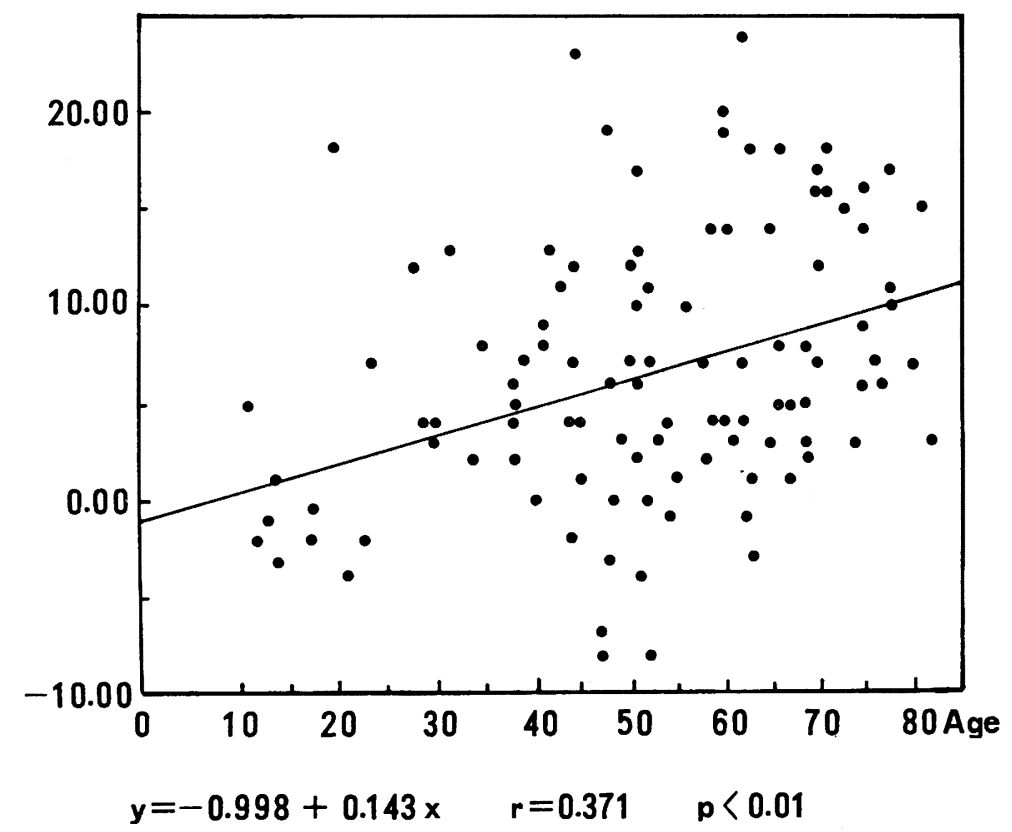

Fig. 4 Relationship between aging in the controls and L : P-L value of the echogenicity index
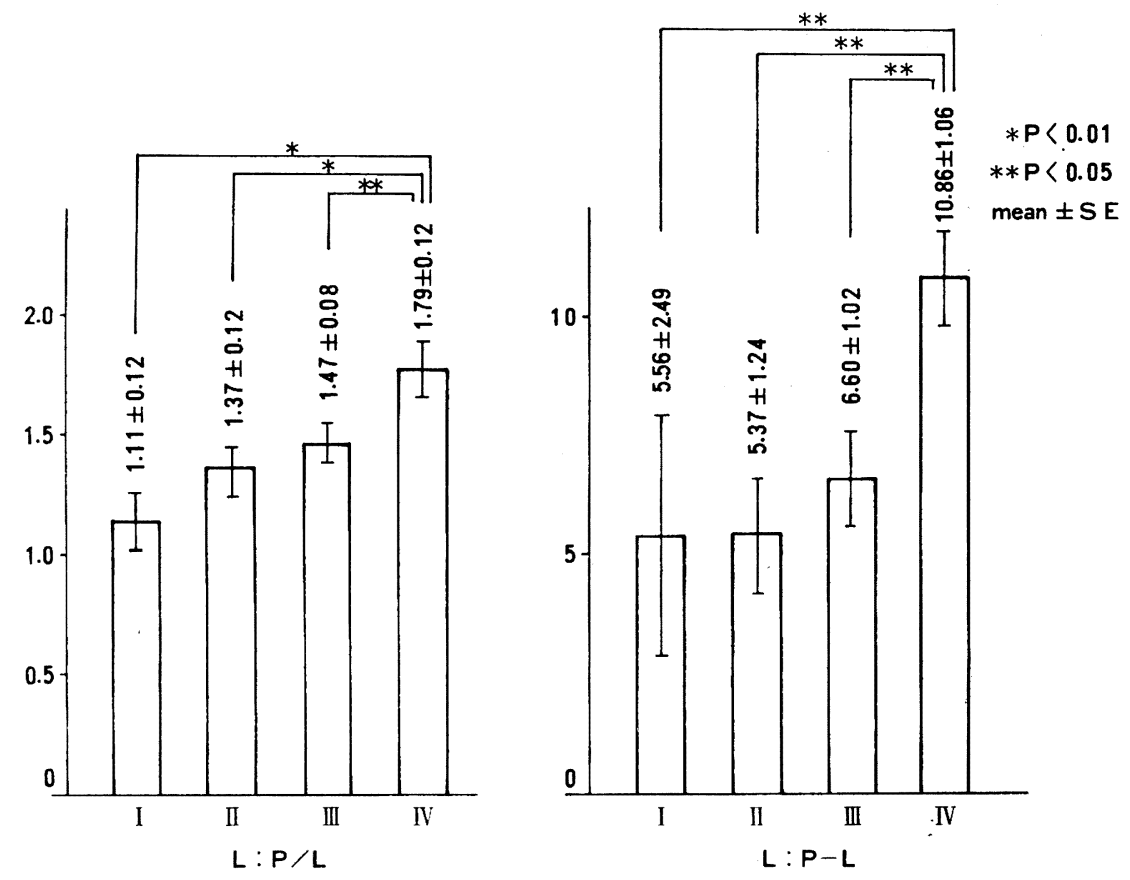

I $: n=13 \quad$ II $: n=30 \quad$ III $: n=49 \quad$ IV $: n=20$

Fig. 5 Influence of aging on $\mathrm{L}: \mathrm{P} / \mathrm{L}$ and $\mathrm{L}: \mathrm{P}-\mathrm{L}$ in normal controls 


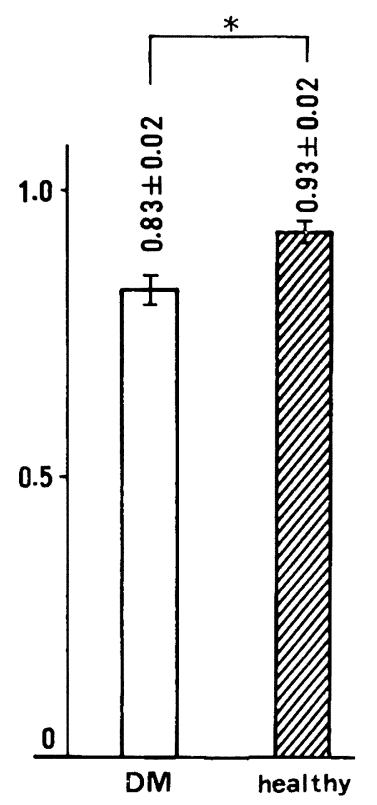

$M: P / L$

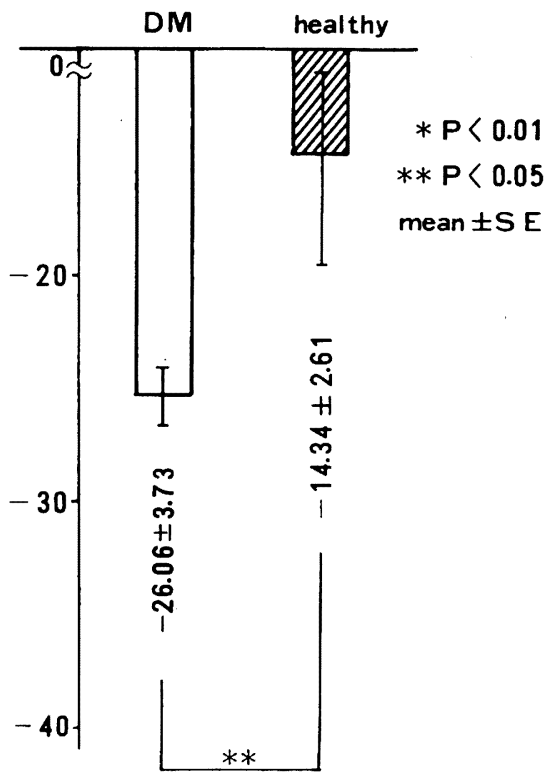

$M: P-L$

$$
\begin{array}{cl}
\text { DM } & : n=56 \\
\text { healthy } & : n=112
\end{array}
$$

Fig. 6 Comparison of $\mathrm{M}: \mathrm{P} / \mathrm{L}$ and $\mathrm{M}: \mathrm{P}-\mathrm{L}$ between the diabetics and the controls

\section{ii) Cases of diabetes mellitus}

Pixel counts were significantly lower in the cases of diabetes mellitus $(M: P / L=0.83$ $\pm 0.02 ; \mathrm{M}: \mathrm{P}-\mathrm{L}=-26.26 \pm 3.73$ ) than in the healthy controls (Fig. 6).

In terms of luminance level $(\mathrm{L}: \mathrm{P} / \mathrm{L}$ and $\mathrm{L}: \mathrm{P}-\mathrm{L})$, no significant difference was noted between diabetics and healthy controls.

When each age group of diabetics was compared with the healthy controls of the corresponding ages, the pixel count was significantly lower in the diabetics aged between 50 and 60 years $(\mathrm{M}: \mathrm{P} / \mathrm{L}=0.82 \pm 0.03 ; \mathrm{M}: \mathrm{P}-\mathrm{L}=-30.04 \pm 5.49)$ than in the healthy controls (Fig. 7).

In terms of echo level $(\mathrm{L}: \mathrm{P} / \mathrm{L}$ and $\mathrm{L}: \mathrm{P}-\mathrm{L})$, no significant difference was noted between any age group of the diabetics and controls.

iii) Analysis in relation to duration of diabetes and presence/absence of complication and insulin therapy

When the diabetics were divided into three groups based on the duration of sickness (less than 1 year, between 2 and 6 years, and over 7 years), there was no significant inter-group 


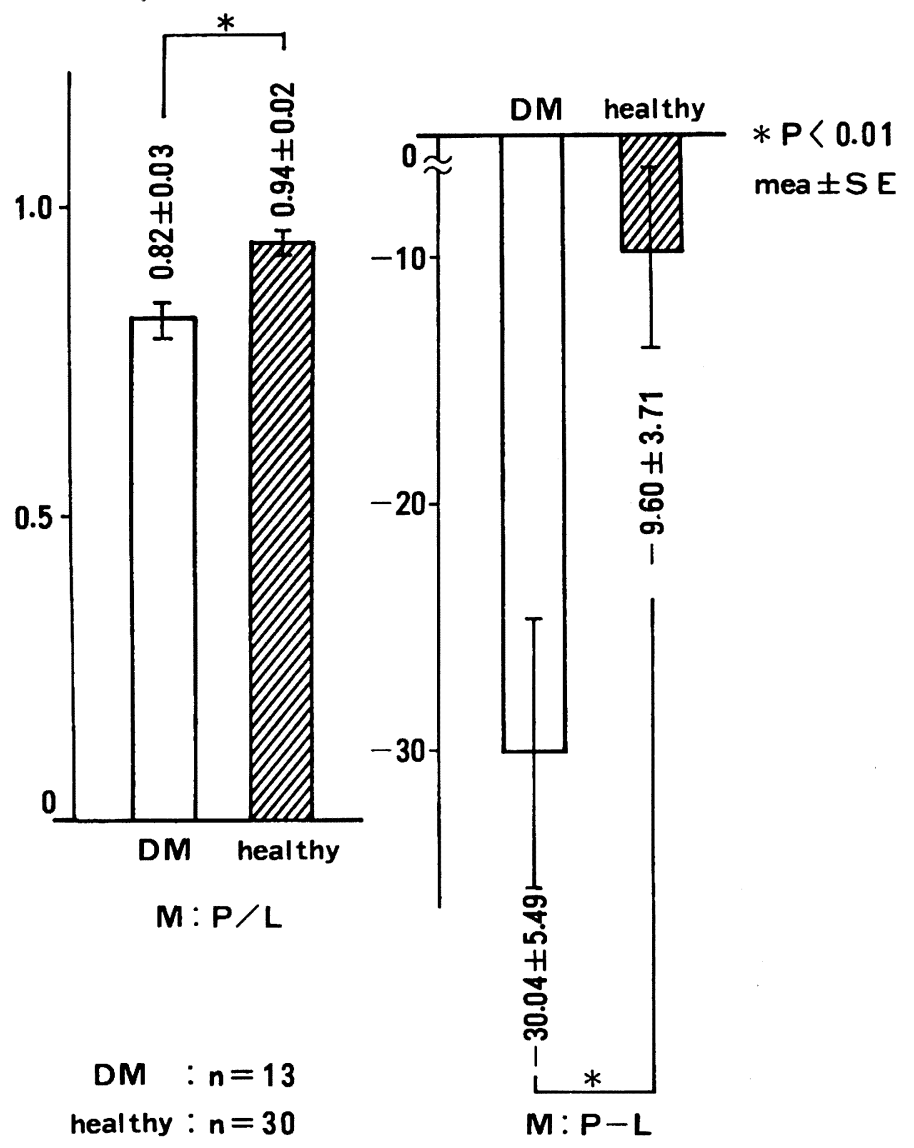

Fig. 7 Comparison of $\mathrm{M}: \mathrm{P} / \mathrm{L}$ and $\mathrm{M}: \mathrm{P}-\mathrm{L}$ between the diabetics and th controls (Group III)

difference in any parameter.

In addition, when the patients were divided according to the presence/absence of complications and insulin therapy, no significant inter-group difference was noted.

The above-mentioned results in the clinical cases can be summarized as in Table 1, which indicates that $\mathrm{M}: \mathrm{P} / \mathrm{L}$ and $\mathrm{M}: \mathrm{P}-\mathrm{L}$ are useful in distinguishing diabetics from healthy individuals, and that $\mathrm{L}: \mathrm{P} / \mathrm{L}$ and $\mathrm{L}: \mathrm{P}-\mathrm{L}$ are useful in assessing the aging-induced changes of the normal pancreas.

2) Autopsied cases

Using pancreas specimens from autopsied cases, the influence of histological changes on the luminance level and pixel count was assessed using water immersion method.

i) Aging-induced changes in normal pancreas

Pancres specimens from 13 autopsied cases without no pancreatic and hepatic disease (aged between 40 and 99 years) were examined (Table 2). Like the afore-mentioned results from 
Table 1 Summary of ultrasound histograms

\begin{tabular}{|c|c|c|c|c|c|}
\hline \multicolumn{2}{|c|}{} & \multicolumn{2}{c|}{$\mathrm{L}$} & \multicolumn{2}{c|}{$\mathrm{M}$} \\
\hline \multicolumn{2}{|c|}{} & $\mathrm{P} / \mathrm{L}$ & $\mathrm{P}-\mathrm{L}$ & $\mathrm{P} / \mathrm{L}$ & $\mathrm{P}-\mathrm{L}$ \\
\hline Healthy aging & & $\mathrm{O}^{1)}$ & $\mathrm{O}^{11}$ & $\times$ & $\times$ \\
\hline \multirow{2}{*}{ DM vs Healthy } & Total & $\times$ & $\times$ & $\bigcirc$ & $\bigcirc$ \\
\cline { 2 - 6 } & Aging & $\times$ & $\times$ & $\mathrm{O}^{2)}$ & $\mathrm{O}^{2)}$ \\
\hline
\end{tabular}

$O$ : statistically significant $(t)$

$X$ : statistically significant (-)

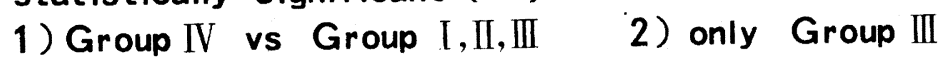

Table 2 Microscopic findings of the autopsied specimen of the normal pancreas \& liver

\begin{tabular}{|r|c|c|c|c|c|c|}
\hline No. & Age & $\mathrm{L}: \mathrm{P} / \mathrm{L}$ & $\mathrm{L}: \mathrm{P}-\mathrm{L}$ & fibrosis & fatty infiltration & $\begin{array}{l}\text { acinar atrophic } \\
\text { changes }\end{array}$ \\
\hline 1 & 40 & 1.86 & 18 & - & + & - \\
2 & 45 & 1.94 & 17 & - & - & - \\
3 & 47 & 1.94 & 17 & + & \pm & - \\
4 & 53 & 1.67 & 12 & + & - & + \\
5 & 53 & 1.9 & 18 & + & + & + \\
6 & 57 & 1.78 & 14 & + & \pm & + \\
7 & 60 & 1.6 & 12 & + & \pm & + \\
8 & 61 & 1.74 & 14 & + & + & + \\
9 & 61 & 2.31 & 11 & + & + & + \\
10 & 64 & 1.76 & 16 & + & + & + \\
11 & 74 & 2.06 & 19 & + & \pm & + \\
12 & 76 & 1.48 & 10 & + & + & + \\
13 & 99 & 2.12 & 19 & + & + & + \\
\hline \multicolumn{7}{|c}{}
\end{tabular}

study, normal pancreas from autopsied cases tended to show an elevation of $\mathrm{L}: \mathrm{P} / \mathrm{L}$ and a clinical L : P-L with age, although no definite correlation of these parameters with age was seen because the number of cases examined was small. In histological examination for evidence of fibrosis, fatty filtration and disturbed acinar structure (due to irregularity in acinar size), fibrosis and disturbed acinar structure became more marked with age. These two changes were severer in Case 12 (a 99-year-old case showing the highest $\mathrm{L}: \mathrm{P} / \mathrm{L}$ and $\mathrm{L}: \mathrm{P}-\mathrm{L}$ ) than in Case 1 (age 44), as shown in Fig. 8-a, b, c. Thus, disturbed acinar structure (which accompanied fibrous hyperplasia and a decrease of cells in the pancreatic lobuli) rather than changes due to fat substitution were more strongly related to aging.

Regarding the distribution of pixels, no histologically characteristic change was found. 

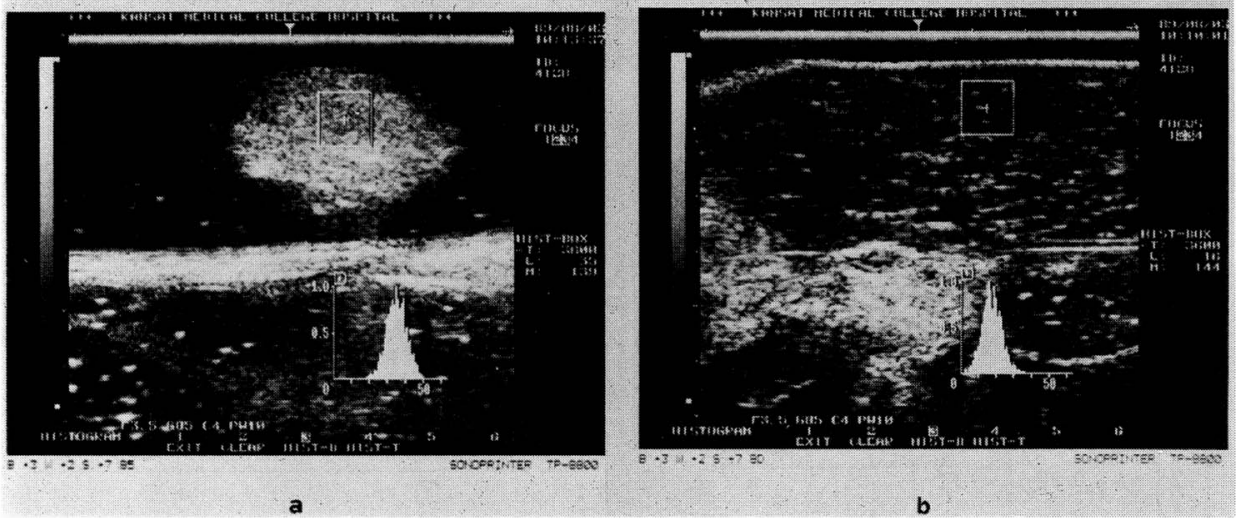

Fig. 8-a, b Water immersion echograms of pancreas (a) and liver (b) in an autopsied control (case 11 on Table 2)

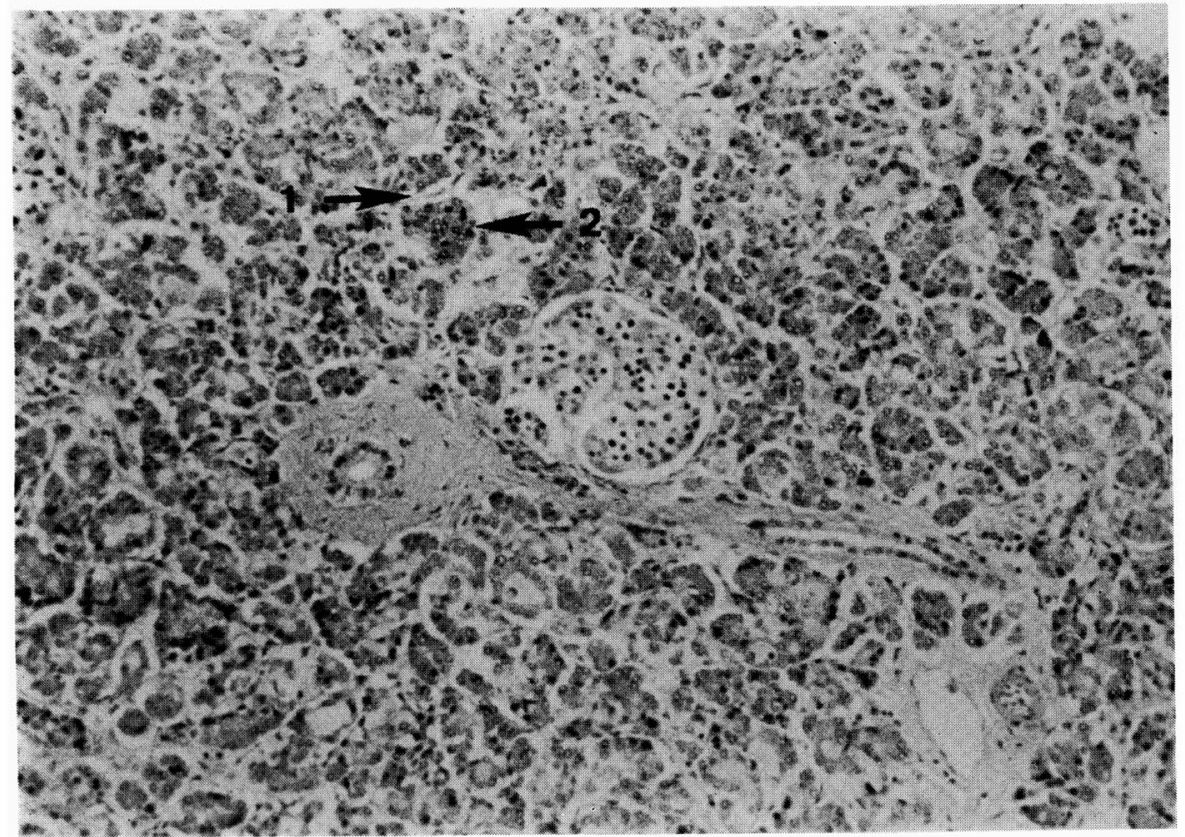

Fig. 8 - c Histology of pancreas showing fibrosis (1) and atrophy of acinor gland (2) in the control case 
Table 3 Microscopic findings of the autopsied spcimen of the diabetes mellitus

\begin{tabular}{|c|c|c|c|c|c|c|c|c|}
\hline No. & Age & $M: P / L$ & $M: P-L$ & fibrosis & fatty & infiltration & acinar at rophic changes & hyalinosis of islets \\
\hline 1 & 53 & 0.78 & -29 & + & & + & + & + \\
\hline 2 & 52 & 0.78 & -31 & H & & + & + & + \\
\hline 3 & 56 & 0.81 & -28 & H & & - & H & H \\
\hline 4 & 64 & 0.81 & -27 & H & & H & H & H \\
\hline 5 & 52 & 0.82 & -24 & H & & + & + & H \\
\hline 6 & 83 & 0.84 & -23 & H & & H & + & H \\
\hline 7 & 76 & 0.85 & -20 & + & & + & + & H \\
\hline 8 & 74 & 0.86 & -21 & + & & H & + & H \\
\hline 9 & 79 & 0.87 & -15 & + & & + & + & + \\
\hline 10 & 84 & 0.87 & -19 & H & & H & + & + \\
\hline 11 & 70 & 0.91 & -12 & + & & H & + & + \\
\hline 12 & 74 & 0.92 & -11 & H & & + & H & + \\
\hline 13 & 71 & 0.94 & -8 & + & & H & H & H \\
\hline 14 & 54 & 0.94 & -7 & H & & + & + & \pm \\
\hline 15 & 69 & 0.98 & -3 & + & & + & + & + \\
\hline 16 & 62 & 0.99 & -1 & H & & $H$ & H & \pm \\
\hline
\end{tabular}

ii) Cases of diabetes mellitus

The results for pancreas specimens from 16 autopsied cases of diabetes mellitus (aged between 52 and 84 years) are shown in Table 3. In this table, cases are arranged in ascending order of $\mathrm{M}: \mathrm{P} / \mathrm{L}$ and $\mathrm{M}: \mathrm{P}-\mathrm{L}$ (which showed a significant inter-group difference in the clinical cases as already mentioned). In histological examination, fibrosis, fatty infiltration, acinar atrophic change and hyalinosis of islet were assessed. As shown in that table, acinar atrophic change and islet degeneration were observed less frequently as $\mathrm{M}: \mathrm{P} / \mathrm{L}$ and $\mathrm{M}: \mathrm{P}-\mathrm{L}$ (indices of pixel count) increased. Islet degeneration or hyalinosis was particularly intense in Cases 3 through 8 (Fig. 9-a, b, c). Fibrosis and fatty infiltration frequently changed corresponding to the ages, and no evident tendency of their aggravation in diabetics was found.

\section{Discussion}

In recent years, attempts have been made to utilize objective evaluation with $\mathrm{B}$-mode pulse echo technique in differential diagnosis of diffuse diseases. Such attempts involve determination of attenuation ${ }^{1-5)}$, determination of acoustic velocity ${ }^{15)},{ }^{16)}$, and histograms of echo levels. Attempts based on histograms of echo levels have include analysis of center of gravity $\left.^{6)}, 7\right)$, analysis of standard deviation ${ }^{8-11)}$, pattern analysis ${ }^{12)},{ }^{13}$ ), and preparation of an echo level map ${ }^{14)}$. However, all such past studies were conducted on superficial organs (thyroid, mammary gland, etc.) and the liver. Studies on the pancreas are scant. 


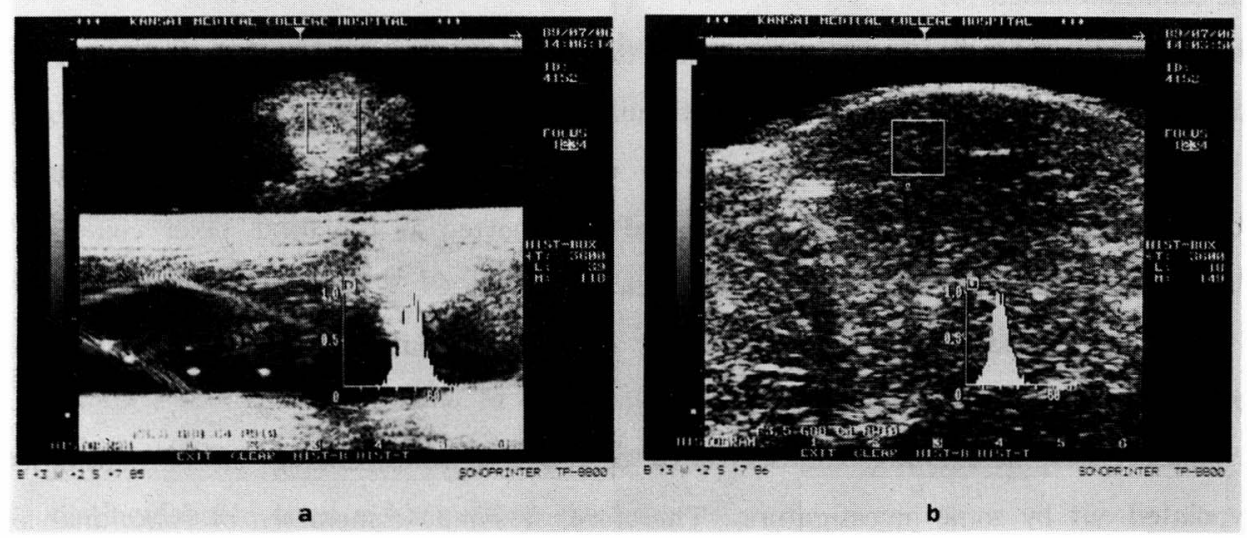

Fig. 9-a, b Water immersion echograms of pancreas (a) and liver (b) in an autopsied diabetic patient (case 4 on Table 3 )

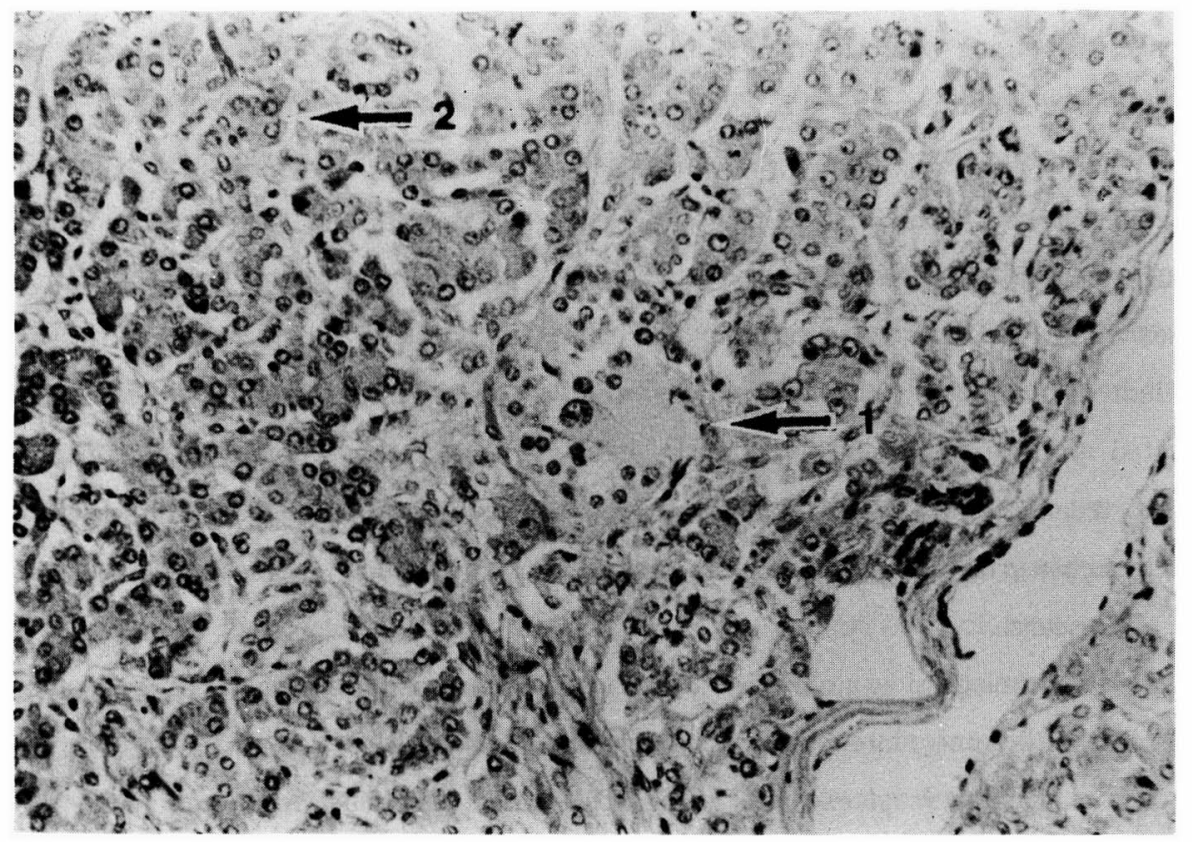

Fig. 9-c Histology of pancreas showing hyalinosis of islet (1) and atrophy of acinor gland (2) diabetic patient 
Furthermore, most past studies involved histogram analysis of echo levels indicative of tissue echo luminance.

Analysis of pixels indicative of tissue echo density is also scant ${ }^{20-22)}$. Nakao et al. ${ }^{20}$ ) compared the total pixel count at the maximum luminance in patients with liver diseases, and found that this comparison allows for assessment of the density of echo pattern. That is, the echo pattern became more coarse and spotted as the total pixel count at the maximum luminance was smaller, typical in cirrhosis cases.

In the present study, I analyzed not only echo levels but also pixels to explore their correlation with histologic changes.

In clinical cases, attenuation and scattering of echo occurs depending on the depth, as has been pointed out by some investigators, Therefore, a simple comparison of echo analysis of ROI in the pancreas (whose depth varies between individuals) is problematic. For this reason, I obtained the dirrerence and ratio of the echo level and pixel count between pancreas and liver.

1) Influence of aging on normal pancreas

In analysis of aging-induced changes in pancreas echo levels in clinical cases, $\mathrm{L}: \mathrm{P} / \mathrm{L}$ and L : P-L (indices of luminance, i. e., internal echo level) were significantly higher. Honjo et al. ${ }^{23)}$, who conducted a histogram analysis of pancreatic echo levels, found a similar age-related increase. One possible factor responsible for this change is fatty infiltration, according to Honjo et al.

Hanada et al. ${ }^{24}, 25$ ) found a positive correlation between $\mathrm{L}: \mathrm{P} / \mathrm{L}$ and age in autopsied elderly individuals (over 65 years), saying that this is histopathologically explained by a complicated mixture of fibrous hyperplasia and fat substitution. Thus, many past studies have reported an elevation of pancreatic echo level with age ${ }^{26-29)}$. The present study supported such a past finding. However, there is no agreed view as to the histologic changes of this echo source.

In our histopathological examination of autopsied pancreas, pancreatic fibrosis and acinar atrophic change became more in tense with age. As a result of irregularity in acinar size, due to decreased numbers of exocrine cells, a disturbed pancreatic acinar arrangement tended to correlate better with aging as compared to changes due to pancreatic fibrosis, although it was not always consistent with an elevation of $\mathrm{L}: \mathrm{P} / \mathrm{L}$ and $\mathrm{L}: \mathrm{P}-\mathrm{L}$. There was a poor correlation between fat substitution and aging.

Sato $^{29)}$ studied aging-related morphological changes in pancreatic exocrine tissue, stroma, fat tissue and islet area, finding that the decrease of exocrine cells, which begins at the age of 70 years, is a major aging-related change, and that the amounts of collagen fibers 
and fat tissue are not aging-related changes. Histological findings supporting the view of Sato were also obtained in this examination of autopsied pancreas, although the number of cases examined was small. In clinical cases, the echo level was significantly higher at ages over 70 than below 70. This finding agrees with Sato's finding that aging-related histological changes occur at ages over 70 .

Therefore, aging-related elevation of pancreatic echo level seems to involve disturbance of pancreatic acinar structure (due to irregularity in size) and fibrosis.

2) Cases of diabetes mellitus

In the clinical study, $\mathrm{M}: \mathrm{P} / \mathrm{L}$ and $\mathrm{M}: \mathrm{P}-\mathrm{L}$ (indices of pixel count) were significantly lower in the diabetics than healthy controls. Particularly in Group III diabetics, this change was clearly distinguishable from aging-related change.

Honjo et al. ${ }^{23}$, who studied echo levels in diabetics, reported that the rate of increase in echo level was higher in diabetics as compared to the aging-induced increase in normal pancreas. On the other hand, Kawagoe et al. ${ }^{30}$ ), who conducted a histogram analysis of echo levels in diabetics, reported the absence of a definite relationship with aging. Also in the present study, the view that the increase of echo level in diabetics is larger than aginginduced increase was not documented, However, pixel count (indicative of density of pancreatic echo) was signigicantly lower in the diabetics than healthy controls of corresponding age groups.

When a decrease of the pixel in diabetics was examined in autopsied cases using ultrasound images, islet degeneration (particularly intense hyalinosis) was linked to the decrease of pixels, while fibrous hyperlasia, fat substituion and disturbed acinar structure were found to not be associated.

Enoki at al. ${ }^{31}$ histologically found that the count of islets per 1 square-centimeter pancreatic body of patients with type II diabetes was $117.2 \pm 67.5$. In the present study, I designated 1 square-centimeter ROI in the pancreatic body, and 3600 of total pixels in this region were evaluated. Therefore, the above-mentioned number of islets seems to be sufficient as a source of echo. If hyalinosis occurs in all these islets, they can serve as a large source of echo. This may lead to a decrease of pixels at the most frequent luminance level and to a less dense pancreatic echo. In some cases (eg. Cases 1 and 2, Table 1), islet degeneration was slight in spite of small pixel count. These cases suggest that a relationship between ultrasound findings from water-immersed pancreas of autopsied cases and histopathological changes is not always found in clinical cases, for the following reasons.

First, examination of fixed specimens from autoposed cases any differs from that of living bodies. Second, in autospied cases, postmortem change is likely to cause autolysis of the 
pancreas, providing different histological features. Third, the position of ROI in the pancreas in water immersion echo technique is not completely consistent with the site of histological examination. Fourth, autopsied cases tend to be elderly; hence, analysis of histogram and histological findings in younger autopsied cases is insufficient. Fifth, the number of autopsied cases avaiable tends to be small, making statistical analysis difficult.

These factors seem to be involved in the inconsistency between the values from histogram and the histological findings in some cases (Table 3). In this sense, more strict evaluation will be necessary.

3) Analysis in relation to duration of diabetes, complications and insulin therapy.

In analysis of the relationship to duration of diabetes and presence/absence of complications and insulin therapy, no significant difference was found. When examined in detail, the timespan from disease onset to diagnosis was inaccurate in many cases; hence, it is not proper to count the duration of sickness always from the time of diagnosis.

In addtiion, the degree of blood glucose control differed between cases under insulin therapy; hence, it is not sufficient to simply compare between cases under insulin therapy and those not, and more detailed examination is necessary with regard to insulin therapy. Analysis in relation to presence/absence of complications revealed no significant difference related to complications. This means that the presence of complications did not lead to abnormal histogram findings due to intense morphological changes of the pancreas.

\section{Conclusion}

Objective assessment of pancreatic acoustic changes based on analysis of histograms (indicative of the distribution of pancreatic echo levels) and pixels was performed. The results of this assesssment was compared with histological findings of autopsied pancreas. The following results were obtained.

1) In normal pancreas, the ratio and difference of the echo luminance level (at the most frequent level) between the pancreas and liver were found to correlate positively with aging. They significantly increased at ages over 70. In histological examination a major aging-induced change was disturbance of acinar structure (due to irregularity in size). In addition, pancreatic fibrosis was also involved in aging-induced changes to the pancreas.

2) The ratio and difference in the pixel count between the pancreas and liver was significantly lower in cases of diabetes mellitus, indicating decreased pixels. This change in pixel count was associated with degeneration of islets (in particular hyalinosis).

3) Duration of diabetes and presence/absence of complications and insulin therapy did not significantly affect the pancreatic echo levels and pixels, although more detailed study is 
necessary in the future.

A summary of this paper was presented at the 19th annual meeting of the Japan Sociaty of Pancreatic Studies and ta the 53rd procceding of the Japan Society of Ultrasonics in Medicine.

\section{Acknowledgment}

Auther is indebted to prof. Sameshima (Third Dept. of Internal Medicine, Kansai Medical University) for her guidance and advice with regard to the manuscript, to Lecturer Uchiyama of the same department for his direct guidance in this experiment, and too Dr. Inui (Second Dept. of Pathology) for his cooperation in autopsy.

\section{References}

1) Itoh, K., Mori, H., Asaoka, Y., Saitoh, T., Suzuki, I0., Taniguchi, N., Yasuda, K., Itoh, H., Itoh, T., Konishi, T. and Koyano, M. : Studies on frequency dependent attenuation with spectralshift zero-crossing (2nd-r). JSUM proc., 50, 201 202, 1987.

2 ) Tanaka, S., Kitamura, T., Yamamoto, K. and Ishikawa, Y. : Estimation of ultrasound attenuation of reflected and transmitted wave. JSUM proc., 50, 281 282, 1987.

3 ) Soetanto, K., Tanaka, M., Ohtsuki, S. and Okujima, M. • Measrement of frequency characteristics of ultrasonic attenuation of rat hepatoma. JSUM proc., 50, 283 284, 1987.

4) Akaiwa, A., Matsuki, T., Kihaile, P. and Maeda, K. : Analysis and imaging of ultrasonic RFsignals. JSUM proc., 50, 329 330, 1987.

5 ) Mori, H., Itoh, K., Taniguchi, N. and Yasuda, K. : Studies on frequency dependent attenuation with spectral-shift zero-crossing (3rd-r.). JSUM proc., 51, 823 824, 1987.

6) Itoh, K., Yasuda, K., Aihara, T., Zhao, L., Konishi, T. and Koyano, A. : Histogram pattern diagnosis using ultrasound images. Jpn. J. Med. Ultrasonics, 10, 292 298, 1983.

7 ) Itoh, K., Yasuda, K., Aihara, T., Koyano, A. and Konishi, T. : Acoustic intensity histogram pattern diagnosis of liver diseases. J. Clin. Ultrasound, 13, 449 456, 1985.

8 ) Usuki, H., Misumi, T., Yoshizawa, J., Hirai, S., Hirai, R., Takeuchi, R., Yokoyama, S., Soga, H., Komatsubara, S. and Teramoto, S. : Evaluation of echo-level histogram patterns with liver tumors. JSUM proc., 50, 237 238, 1987.

9) Usuki, H., Hirai, S., Yoshizawa, J., Hirai, R., Yokoyama, S., Komatsubara, S. and Teramoto, S. : Evaluation of echo-level histogram pattern with liver tumors. JSUM proc., 51, 825 826, 1967.

10) Kishimoto, Y. and Fujiki, Y. : Ultrasonic image analysis of liver hemangioma and hepato Cellular Carcinoma. JSUM proc., 57, 57 58, 1987.

11) Tanaka, T., Sugiyama, Y., Hanazawa, K., Ohuchi, T. and Kitagawa. R. : Initial trial of echo pattern digital expression used by echo-histogram. JSUM proc., 51, 252 253, 1987.

12) Kurita, T., Katoh, S. and Endoh, M. : Ultrasonic echo level and histogram in thyroid diseases. JSUM proc., 50, 199 200, 1987.

13) Nishi, K. and Tatsuno, I. : Evaluation of echo histogram with liver using ultrasonic images. Sakura $\mathrm{X}$ ray photograph research, 37, 3 6, 1986.

14) Mallioux, G. E. , Bertrand, M. and Stampfler, R. : Local histogram information content of ultrasound b-mode echographic texture. Ultrsound in MED. \& Biol., 11, 743 750, 1985. 
15) Akamatsu, K., Miyauchi, S., Nishimura, Y. and Ohta, Y. : A study on the cause of increase in vivo hepatic ultrasound velocity in the cirrhotic liver measured crossed beam method JSUM proc., 50, 235 236, 1987.

16) Nishimura, Y., Miyauchi, S., Akamatsu., K., Ohta, Y., Okazaki, K., Hirata, M. and Iinuma, K. : A study on non-invasive quantitative assessment of fatty metamorphosis in the liver. JSUM proc., 50, 233 234, 1987.

17) Takada, E., Ishizuka, H., Fukushima, Y., Morikubo, H., Ikeguchi, S. and Shida, N. : Inf luence of abdominal wall to the ultrasonogram. JSUM proc., 50, 277 278, 1987.

18) Sou, K., Itoh, T. and Konishi, T. : A new method for amplitude histogram analysis in B-mode ultrasonography. JSUM proc., 51, 171 172, 1987.

19) Kimura, S. : Quantitative estimation of liver-kidney contrast using echo-histogram analyiss in diffuse liver diseases and renal diseases. Jpn. J. Med. Ultrasonics, 13, 425 433, 1986.

20) Nakao, K., Iwata, K., Takeuchi., K. and Murashima, N. : Quantitative evaluation of the intrahepatic echo pattern. ISUM proc., 51, 827 828, 1987.

21) Sommer, F. G., Joynt, L. F., Carroll, B. A. and Macovski, A. : Ultrasonic characterization of abdominal tissues via digital analysis of backscatterd waveforms. radiology, 141, 811 817, 1981.

22) Zagzebski, J. A., Madsen, E. L. and Goodsitt, M. M. : Quantitative tests of three-dimensional gray scale texture model. ultrasonic imaging, 7, 252 263, 1985.

23) Honjyo, N., Watanabe, K., Miura, T., Nakanishi, T., Nakano, T., Inoue, T., Hayashi, H., Kawamura, T. and Ogawa, H. : Sonographic objective assessment of echo-level in the liver and pancreas using histogram. JSUM proc., 46, 645 646, 1985.

24) Handa, M., Ohdaira, C., Kobari, M., Koizumi, M., Gotoh, Y., Wakamatsu, H. and Komatsu, K. : Quantitative estimation of pancreatic echogenicity and histopathological study of the postmortem pancreas in the elderly. JSUM proc., 50, 435 436, 1987.

25) Hanada, M., Koizumi, M., Ohdaira, C., Shishido, T., Kobari, M., Gotoh, Y., Wakamatsu, H. and Komatsu, K. : Quantitative estimation of pancreatic echogenicity and histopathological study of the postmortem pancreas in the elderly. Jpn. J. Gastroenterol, 85, 2687 2891, 1988.

26) Kataoka, Y., Kashima, T., Horii, Y., Kinugasa, K., Inada, Y., Morinaga, S. and Tatso, T. : ltrasonographic changes of the pancreas with Aging. Jpn. J. Gastroenterol. 79, 1977 1985, 1982.

27) Marks, W. M., Filly, R. A. and Callen, P.W. : Ultrasonic evaluation of normal pancreatic echogenicity and its relationship to pat deposition. Radiology. 137, 475 479, 1980.

28) Aoki, H., Nagoya, K., Nakaxama, K., Sakakura, M. and Matsuzaki, S. A. : A quantitative study of the echo levels of the pancreas in normal subjects. JSUM proc., 50, 437 438, 1987.

29) Satoh, C. : Morphological changes of human pancreas in aging process. The Biliary Tract \& Pancreas, 3, 1243 1249, 1982.

30) Kawagoe, M., Oohashi, H., Iseki, K., Kawabata, Y. and Hirata, Y. : Ultrasonographic objective assessment of echo-level in the pancreas of diabetics. JSUM proc., 48, 715 716, 1986.

31) Enoki, N., Yoshimitsu, M. and Murata, Y. : Quantitative changes of pancreatic islets of the noninsulin dependent diabetes mellitus (NIDDM). Endocrinology. 33, 1071 1075, 1985. 